\title{
GENETIC ALGORITHM FOR SOLVING DYNAMIC SIMULTANEOUS ROUTE AND DEPARTURE TIME EQUILIBRIUM PROBLEM
}

\author{
Shu-Guang Li \\ School of Information Engineering, Chang'an University, Xian 710049, \\ People's Republic of China \\ E-mail:shgli@chd.edu.cn,lxlsg@sohu.com
}

Received 20 September 2007, accepted 20 November 2007

\begin{abstract}
We present a genetic algorithm for solving dynamic simultaneous route and departure time equilibrium problem. Not only can a flow-swapping process in the algorithm guarantee the flow conservation constraints between OD pair, but also accelerate the convergence velocity of the algorithm. Finally, a simulation example shows feasibility and validity of genetic algorithm.
\end{abstract}

Keywords: genetic algorithm, route choices, departure time choices.

\section{Introduction}

The observations show the travelers do adjust routes and departure times to avoid peak period congestion (Hendrickson and Plank 1984). Furthermore, most existing studies (Arnott et al. 1990; Friesz et al. 1993; Wie et al. 1995; Huang and Lam 2002; Szeto and Lo 2004; Lin and Heydecker 2005) focus on dynamic simultaneous route and departure time problem. Many researchers used various models such as simulation and analytical models, and propose some algorithms for dynamic SRD problem. The algorithms are classified into two classes, class I can be described as the strict mathematical algorithm such that Szeto and Lo (2004) adopted Han and Lo (2003) decent direction method to solve the VIP; class II is heuristic algorithm based on flow-swapping rules (Wie et al. 1995; Huang and Lam 2002; Lin and Heydecker 2005). The basic idea of the algorithm is: for each OD pair, inflows on the non-cheapest time-dependent paths are moved to the cheapest paths. Due to non-monistic of the path travel cost function, the above algorithms only can converge to local optimal solution. In order to obtain global optimal solution, Sadek et al. (1997) used a genetic algorithm for dynamic traffic assignment problems; however, the algorithm is based on link. In this paper, we combine genetic algorithm with flow-swapping rules for solving a dynamic simultaneous route and departure time problem.

This paper includes the following several sections, firstly we describe a dynamic simultaneous route and departure time model, then we present a variational in- equality formulation for SRD problem. In section 3, we propose a combined genetic algorithm for the proposed model. Section 4 provides a numerical example to demonstrate and verify the proposed solution algorithms.

\section{Model formulation}

Here, the dynamic network models of Freisz et al. (1993), Chabini (2001) are given as follows:

$u_{a p}^{r s}(k)$ - the inflow rate of travelers on link $a$ of path $p$ during interval $k$.

$v_{a p}^{r s}(k)$ - the departure rate of travelers on link $a$ of path $p$ during interval $k$.

$x_{a p}^{r s}(k)$ - the vehicle numbers of travelers on link $a$ of path $p$ at interval $k$.

$V_{a p}^{r s}(k)$ - the cumulative departures of travelers on link $a$ of path $p$ until interval $k$.

$U_{a p}^{r s}(k)$ - the cumulative arrivals of travelers on link $a$ of path $p$ until interval $k$.

$t_{a}(k)$ - the travel time on link $a$ for travelers entering this link at interval $k$.

The link dynamics equations express the relationship between the flow variables of a link

$$
\begin{aligned}
& x_{a p}^{r s}(k)=x_{a p}^{r s}(k-1)+u_{a p}^{r s}(k)-v_{a p}^{r s}(k) \\
& \forall k, \forall r s, \forall a \in p .
\end{aligned}
$$


The flow conservation equations for the node:

$$
\begin{aligned}
& u_{a p}^{r s}(k)= \begin{cases}f_{p}^{r s}(k) & \text { a is first link on path } p \\
v_{b p}^{r s}(k) & \text { a is after } b\end{cases} \\
& \forall k, \forall r s, \forall p .
\end{aligned}
$$

Flow propagation constraints are used to describe the flow progression over time:

$$
V_{a p}^{r s}(k)=\sum_{j \in[l: 0 \leq j \cdot \delta+T(j) \leq(k-1) \cdot \delta]} u_{a p}^{r s}(j) \cdot \delta \quad \forall k, \forall r s, \forall p .
$$

Definitional constraint:

$$
\begin{aligned}
& x_{a}(k)=\sum_{r s} \sum_{p}\left(x_{a p}^{r s}(k)\right) \quad v_{a}(k)=\sum_{r s} \sum_{p}\left(v_{a p}^{r s}(k)\right), \\
& u_{a}(k)=\sum_{r s} \sum_{p}\left(u_{a p}^{r s}(k)\right) \quad V_{a p}^{r s}(k)=\sum_{j=1}^{k}\left(v_{a p}^{r s}(j)\right) \cdot \delta, \\
& U_{a p}^{r s}(k)=\sum_{j=1}^{k}\left(u_{a p}^{r s}(j)\right) \cdot \delta .
\end{aligned}
$$

Boundary conditions:

$$
\begin{aligned}
& x_{a p}^{r s}(0)=0, u_{a p}^{r s}(0)=0, v_{a p}^{r s}(0)=0, \\
& x_{a p}^{r s}(0)=0, u_{a p}^{r s}(0)=0, v_{a p}^{r s}(0)=0 .
\end{aligned}
$$

Now we give the actual path travel time and actual path travel cost functions. The actual travel time to traverse path $p=\left\{a_{1}, a_{2}, \ldots, a_{n}\right\}$ for travelers entering into the network during interval $k$ is calculated using the following nested function:

$$
\begin{aligned}
& t_{p}^{r s}(k)=t_{a 1}(k)+t_{a 2}\left(k+t_{a 1}(k)\right)+ \\
& \cdots+t_{a m}\left(k+t_{a 1}+\cdots+t_{a m-1}\right),
\end{aligned}
$$

where, let $t_{a 1}=t_{a 1}(k), t_{a 2}=t_{a 2}\left(k+t_{a 1}(k)\right)$ for short.

The schedule delay cost function can be expressed as follows:

$$
\begin{aligned}
& S c h_{s}= \\
& \left\{\begin{array}{l}
\beta\left[t_{s}-\Delta_{s}-k-t_{p}^{r s}(k)\right] \text { if } t_{s}-\Delta_{s}>k+t_{p}^{r s}(k), \\
\gamma\left[k+t_{p}^{r s}(k)-t_{s}-\Delta_{s}\right] \text { if } t_{s}+\Delta_{s}<k+t_{p}^{r s}(k), \\
0 \quad \text { otherwise. }
\end{array}\right.
\end{aligned}
$$

Denote $\left[t_{s}-\Delta_{s}, t_{s}+\Delta_{s}\right]$ as the desired time interval for arrival at the destination $s$ in the network. Where $t_{s}-\Delta_{s}$ is the travelers' desired earliest arrival time, $t_{s}+\Delta_{s}$ is the desired latest arrival time at the destination $s . \beta, \gamma$ is the unit cost of schedule delay (early, late) at the destination $s$, respectively.

Therefore, the travel cost of a trip from origin $r$ to destination $s$ on path $p$ for a traveler leaving origin at time interval $k$ is:

$$
c_{p}^{r s}(k)=\alpha t_{p}^{r s}(k)+S c h_{s}(k)
$$

Where $\alpha$ is a convention factor to transform the path travel time into travel cost. In accordance with the empirical results, we assume that $\gamma>\alpha>\beta$ holds.

Travelers follow dynamic simultaneous path and departure time equilibrium (UE-SRD), expressed as:

$$
\begin{aligned}
& c_{p}^{r s}\left(k, f^{*}\right)\left\{\begin{array}{l}
=c_{\min }^{r s} \text { if } f_{p}^{r s^{*}}(k)>0 \\
>c_{\min }^{r s} \text { if } f_{p}^{r s^{*}}(k)=0
\end{array} \quad \forall r s, k, p .\right. \\
& \sum_{p} \sum_{k} f_{p}^{r r^{*}}(k)=q^{r s} / \delta \quad \forall r s . \\
& f_{p}^{r s^{*}}(k) \geq 0 .
\end{aligned}
$$

Where $c_{\min }^{r s}(\cdot)$ is the minimum unit travel cost of travelers between origin $r$ and destination $s$, $c_{\text {min }}^{r s}(\cdot)=\min \left\{C_{p}^{r s}(k, \cdot), \forall p, k\right\}$. Equation (10) represents the flow conservation of travelers between origin $r$ and destination $s$ and equation (11) represents the non-negativity of all path inflow rates.

For travelers and for each origin-destination (OD) pair, the path travel costs experienced for travelers, regarding of departure times, is equal and minimum, and less than (or equal to) the path travel costs for travelers on any unused paths.

The above UE-SRD equilibrium condition of travelers can be expressed by a finite dimensional variational inequality formulation.

Find a vector $f^{*} \in \Omega$ if and only if it satisfies:

$$
\sum_{r s} \sum_{p} \sum_{k} c_{p}^{r s^{*}}\left(k, f^{*}\right)\left(f_{p}^{r s}(k)-f_{p}^{r s^{*}}(k)\right) \geq 0, f \in \Omega .
$$

Where, $\Omega$ is a closed convex:

$$
\hat{\Omega}=\left\{\hat{f} \mid \sum_{p} \sum_{k} \hat{f}_{p}^{r s}(k)=\frac{\hat{q}^{r s}}{\delta}, \hat{f}_{p}^{r s}(k) \geq 0, \forall r s\right\} .
$$

\section{Algorithm}

GAs are search and optimization procedures motivated by natural principles and selection. Because of their simplicity, minimal problem restrictions, global perspective, and implicit parallelism, GAs have been applied to a wide variety of problem domains including engineering, sciences, and commerce (Yin 2000). In this section, we propose a genetic algorithm for solving VIP(12), which mainly include several parts: determination of the initial population, implementation of the constraints, convergence indicator, fitness function, and the crossover and mutation operation, etc.

\subsection{The initial population}

A population of chromosomes is initialized by the following equation:

$$
f_{p}^{r s}(k)=\frac{q^{r s}}{K \cdot\left|P_{r s}\right|} \cdot R n d,
$$

$R n d$ expresses the random count between $[0,1] ; K$ represents total time interval; $\left|P_{r s}\right|$ represents the path 
number between OD pair $r$. The initial path inflow cannot follow the flow conservation constraint (10).

\subsection{Implementation of the constraints}

The implementation of constraints of VIP(12) is an important question to be considered both in initializing a population and designing an objective evaluation function. The implementation of constraints can be classified into two classed: one is by imposing moderate penalties on individuals that violate them, the other is by creating individuals directly satisfying them by means of a decoding procedure or decoder. In this paper, we propose flow equilibrium decoders that avoid the violation of the flow conversation constraint. A decoder is similar with the flow swapping processes given by Wie et al. 1995, Huang, Williams 2002.

The population that violate the flow conservation constraint can be classified into two conditions, one is $\sum_{p, k} f_{p}^{r s^{*}}(k)<q^{r s}$, the other is $\sum_{p, k} f_{p}^{r s^{*}}(k)>q^{r s}$. Here, the path travel cost $c_{p}^{r s^{*}}(k)$ is obtained by dynamic network loading process (Chabini 2001) according to $f_{p}^{r s^{*}}(k)$, if $\sum_{p, k} f_{p}^{r s}(k)<q^{r s}$.

If the sum of the path inflow rate of an individual is less than actual OD demand for each OD pair rs. $q^{r s}-\sum_{p, i} f_{p}^{r s^{\star}}(i)$ is moved to the cheapest time-dependent path. The equation can be expressed as follows:

$$
f_{p}^{r s^{* *}}(k)=f_{p}^{r s^{*}}(k)+\frac{q^{r s}-\sum_{p, i} f_{p}^{r s^{*}}(i)}{\left|P_{\min }^{r s}\right|},(p, k) \in P_{\min }^{r s},(
$$

$P_{\min }^{r s}$ expresses the cheapest time-dependent path set.

$$
\text { If } \sum_{p, k} f_{p}^{r s^{*}}(k)>q^{r s} \text {. }
$$

If the sum of the path inflow rate of an individual is more than actual OD demand for each OD pair $r s$. The inflows on the non-cheapest time-dependent path are subtracted according to a proportion in order to guarantee the satisfaction of the flow conservation constraint. The equations can be expressed as follows:

$$
\begin{aligned}
& f_{p}^{r s^{* *}}(k)=\max \left\{0, f_{p}^{r s^{*}}(k)-\right. \\
& \left.\eta \cdot f_{p}^{r s^{*}}(k) \cdot\left(c_{p}^{r s^{*}}(k)-c_{\min }^{r r^{*}}+\varepsilon\right)\right\} . \\
& \sum_{p, k} f_{p}^{r r^{* *}}(k)=q^{r s} .
\end{aligned}
$$

The volumes subtracted are proportional to $\eta \cdot f_{p}^{r s^{*}}(k) \cdot\left(c_{p}^{r s^{*}}(k)-c_{\min }^{r s^{*}}+\varepsilon\right) \cdot \eta$ represents the flow adjusting parameter that is calculated by one dimension search method based on Eqs. (15-16). $\varepsilon$ is a given cheapest timedependent path flow adjusting parameter, in order to avoid the sum of inflows on the cheapest time-dependent path being more than actual OD demand, the value of the parameter can be set as a very small number.

Not only can a flow-swapping process in the algorithm guarantee the flow conservation constraints bet- ween OD pairs, but also accelerate the convergence velocity of the algorithm.

\subsection{Convergence indicator}

The convergence indicator of chromosome $i$ at iteration $k$ can be expressed as follows:

$$
\rho_{i}^{t}=\frac{\sum_{r s, p, k} f_{p}^{r s}(k)\left(c_{p}^{r s}(k)-c_{\min }^{r s}\right)}{\sum_{r s, p, k} f_{p}^{r s}(k) c_{\min }^{r s}} .
$$

The convergence indicator of chromosomes can be calculated by the following equation:

$$
\rho_{i, \min }=\min \left\{\rho_{i}^{t}, \forall t\right\} .
$$

\subsection{Fitness function}

We define the fitness function as:

$$
F_{i}^{t}=\alpha \cdot \frac{1}{\rho_{i}^{t}},
$$

where $\alpha$ is the accelerating parameter of the algorithm; en below:

The step-by-step procedure of our algorithm is giv-

- Step 1: Select at random the initial population, crossover and mutation probability.

- Step 2: Flow equilibrium decoder.

- Step 3: Calculate the fitness functions for individuals by (19)

- Step 4: convergence.

If $\rho_{i, \min }<\varepsilon, i=M$, stop, otherwise.

- Step 5: Reproduce a new population $(n+1)$ from population $n$ according to the distribution of fitness function.

- Step 6: Genetic Operators.

- Crossover: Cross two individuals chosen from population $(n+1)$ with a specified crossover probability, $p c$.

- Mutation: Select one individual from population $(n+1)$ with a specified probability, pm.

Go to step 2.

\section{Numerical example}

The numeral example is a TF network as shown in Fig. 1. It consists of 13 nodes, 19 links and two OD pairs $((1.11),(3.13))$. The link parameter is shown in Table 1,

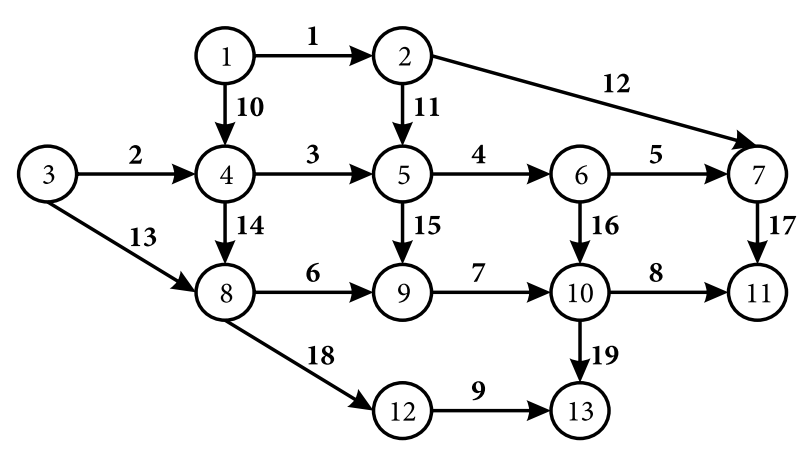

Fig. 1. The example network 
Table 1. Link parameter

\begin{tabular}{|c|c|c|c|}
\hline Link number & Start node & End node & Link free flow time \\
\hline 1 & 1 & 2 & 0.020 \\
\hline 2 & 3 & 4 & 0.016 \\
\hline 3 & 4 & 5 & 0.024 \\
\hline 4 & 5 & 6 & 0.010 \\
\hline 5 & 6 & 7 & 0.024 \\
\hline 6 & 8 & 9 & 0.026 \\
\hline 7 & 9 & 10 & 0.016 \\
\hline 8 & 10 & 11 & 0.010 \\
\hline 9 & 12 & 13 & 0.014 \\
\hline 10 & 1 & 4 & 0.014 \\
\hline 14 & 4 & 8 & 0.026 \\
\hline 11 & 2 & 5 & 0.028 \\
\hline 15 & 5 & 9 & 0.028 \\
\hline 16 & 6 & 10 & 0.012 \\
\hline 17 & 7 & 11 & 0.010 \\
\hline 19 & 10 & 13 & 0.012 \\
\hline 12 & 2 & 7 & 0.010 \\
\hline 13 & 3 & 8 & 0.016 \\
\hline 18 & 8 & 12 & 0.018 \\
\hline & & & \\
\hline
\end{tabular}

Table 2. Path link series

\begin{tabular}{|c|c|c|}
\hline OD & Path & Link \\
\hline \multirow{5}{*}{$(1,11)$} & Path 1 & $1-12-17$ \\
& Path 2 & $1-11-4-5-17$ \\
& Path 3 & $1-11-4-16-8$ \\
& Path 4 & $1-11-15-7-8$ \\
& Path 5 & $10-3-4-5-17$ \\
& Path 6 & $10-3-4-16-8$ \\
& Path 7 & $10-3-15-7-8$ \\
& Path 8 & $10-14-6-7-8$ \\
\hline \multirow{5}{*}{$(3,13)$} & Path 1 & $13-18-9$ \\
& Path 2 & $2-14-18-9$ \\
& Path 3 & $13-6-7-19$ \\
& Path 4 & $2-3-4-16-19$ \\
& Path 5 & $2-3-15-7-19$ \\
& Path 6 & $2-14-6-7-19$ \\
\hline
\end{tabular}

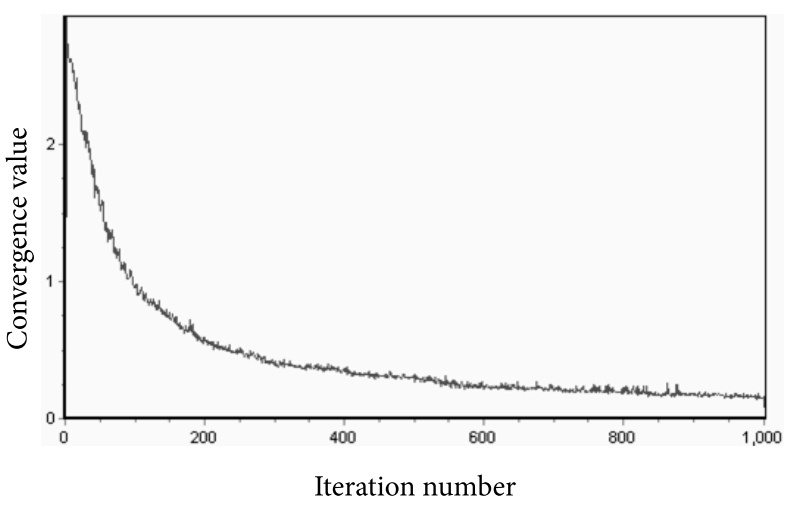

Fig. 2. Convergence of the Gas

the path link series is shown in Table 2. Other input data are: $\alpha=6.4 ; \beta=3.9 ; t_{s}=9.0 ; \Delta_{s}=0.25 ; \varepsilon=0.00001$, set $T$ be from 6 to 10.A.M. and $K=100$. The proposed solution algorithm was coded in Visual Basic 6.0, and run on a personal computer (P4 2.88G).

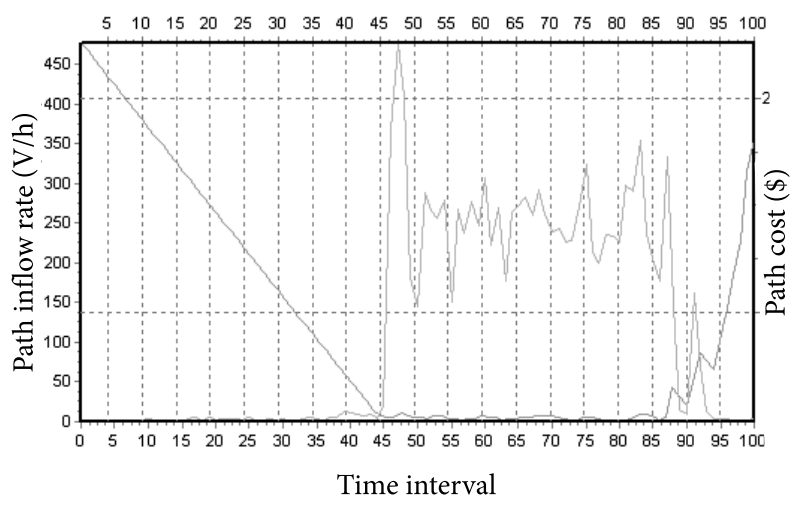

Fig. 3. Path inflow rate and cost between path 1 of OD pair $(1,11)$

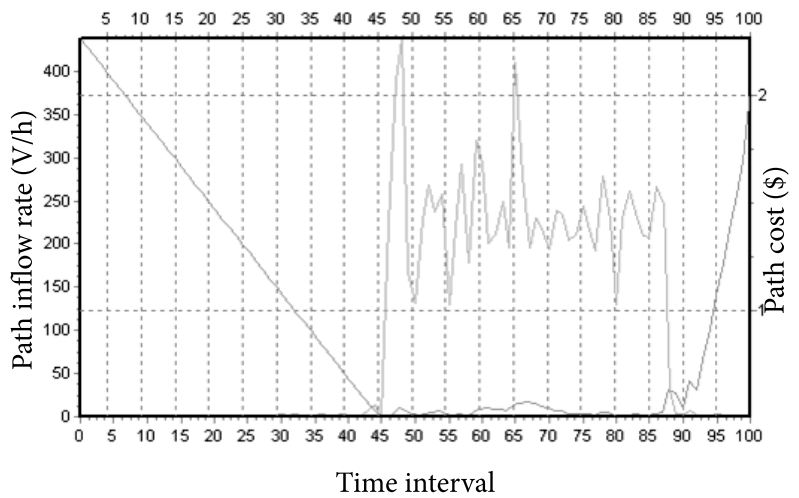

Fig. 4. Path inflow rate and cost between path 1 of OD pair $(3,13)$

The value of the convergence indicator of the algorithm is decreasing as the iteration number increases as shown in Fig. 4. When the iteration number amounts to 1000 , the value of the convergence indicator descends to 0,09 . Figs. 4,5 give the path inflow rates and the travel costs on path 1 of OD pairs $(1,11)$ and path 3 of OD pair $(3,13)$. We can find an approximate dynamic equilibrium pattern for all route inflow rates of travelers between two OD pairs. The other path flow and cost on network have similar dynamic equilibrium conditions. Due to limitation of page, we don't give all path flow patterns.

\section{Conclusion}

We propose a genetic algorithm for solving dynamic simultaneous route and departure time equilibrium problem. Not only can a flow equilibrium decoder in the algorithm guarantee the flow conservation constraints between OD pair, but also accelerate the convergence velocity of the algorithm. Finally, a simulation example shows feasibility and validity of genetic algorithm. In future studies, the genetic algorithm is compared with the other algorithms such as flow-swapping algorithm, VI algorithm and so on. And a simulation example is given in real network. 


\section{References}

Arnott, R.; de Palma, A.; Lindsey, R. 1990. Departure time and route choice for the morning commute, Transportation Research Part B: Methodological 24B(3): 209-228.

Chabini, I. 2001. Analytical dynamic network loading problem: formulation, solution algorithms, and computer implementations, Transportation Research Record 1771: 191-200.

Friesz, T. L.; Bernstein, D.; Smith, T. E.; Tobin, R. L.; Wie, B.-W. 1993. A variational inequality formulation of the dynamic network user equilibrium problem, Operations Research 41(1): 179-191.

Han, D.; Lo, H. K. 2004. Solving non-additive traffic assignment problems: a decent methods for co-coercive variational inequalities, European Journal of Operational Research 159(3): 529-544.

Hendrickson, C.; Plank, E. 1984. The flexibility of departure times for work trips, Transportation Research 18A(1): 25-36.

Huang, H.-J.; Lam, W. H. K. 2002. Modeling and solving the dynamic user equilibrium route and departure time choice problem in network with queues, Transportation Research Part B: Methodological 36(3): 253-273.

Yin, Y. 2000. Genetic-algorithms-based approach for bilevel programming models, Journal of Transportation Engineering 126(2): 115-120.

Lin, Y.; Heydecker, B. 2005. Dynamic departure time and stochastic user equilibrium assignment, Transportation Research Part B: Methodological 39(2): 97-118.

Sadek, A. W.; Smith, B. L.; Demetsky, M. J. 1997. Dynamic traffic assignment genetic algorithms approach, Transportation Research Record 1588: 95-103.

Szeto, W. Y.; Lo, H. K. 2004. A cell-based simultaneous route and departure time choice model with elastic demand, Transportation Research Part B: Methodological 38(7): 593-612.

Wie, B.-W.; Tobin, R. L.; Friesz, T. L. 1995. A discrete time, nested cost operator approach to the dynamic network user equilibrium problem, Transportation Science 29(1): 79-92. 